\title{
A fast one dimensional total variation regularization algorithm
}

\author{
A. Makovetskii ${ }^{1}, \mathrm{~S}$. Voronin $^{1}$, V. Kober ${ }^{1}$ \\ ${ }^{1}$ Chelyabinsk State University, ul. Bratiev Kashirinykh, 129, 454001, Chelyabinsk, Russia
}

\begin{abstract}
Denoising has numerous applications in communications, control, machine learning, and many other fields of engineering and science. A common way to solve the problem utilizes the total variation (TV) regularization. Many efficient numerical algorithms have been developed for solving the TV regularization problem. Condat described a fast direct algorithm to compute the processed 1D signal. In this paper, we propose a variant of the Condat's algorithm based on the direct 1D TV regularization problem. The usage of the Condat's method with the taut string approach leads to a clear geometric description of the extremal function.
\end{abstract}

Keywords: Image restoration; total variation; denoising; exact solutions

\section{Introduction}

One of the most known techniques for denosing of noisy signals and images was proposed by Rudin, Osher, and Fatemi [1]. This is a total variation (TV) regularization problem. Let $J(u)$ be the following functional in the functional space $\mathrm{L}_{2}$ :

$$
J(u)=\left\|u-u_{0}\right\|_{L_{2}}^{2}+\lambda T V(u),
$$

where $\left\|u-u_{0}\right\|_{L_{2}}^{2}$ is called a fidelity term and $\lambda T V(u)$ is called a regularization term. Here $u_{0}$ is an observed signal that is distorted by additive noise $n$,

$$
u_{0}=v+n
$$

Consider the following variational problem:

$$
u_{*}=\arg \min _{u \in B V(\Omega)} J(u) .
$$

where $u_{*}$ is an extremal function for $J(u)$. Numerical results have shown that TV regularization is quite useful in image restoration [2-4]. Here we consider a one dimensional TV (1D TV) regularization problem. In [5,6] Strong and Chan considered the behavior of explicit solutions to the 1D TV problem when the parameter $\lambda$ in Eq. (1) is sufficiently small. The exact solutions to one dimensional TV regularization problem and to two dimensional radial symmetric TV regularization problem were considered in [7-10]. Recently, Condat [11,12] proposed explicit solutions to the 1D TV problem as well as a direct fast algorithm for the case of discrete functions. The algorithm is very fast and has complexity of $O(n)$ for typical discrete functions. In contrast, the proposed approach for finding exact solutions has a clear geometrical meaning.

In this paper, we propose a variant of the Condat's algorithm based on the direct 1D TV regularization problem. The usage of the Condat's method with the taut string method [12] leads to a clear geometric description of the extremal function.

\section{Formulation of 1D TV regularization as a discrete problem}

Let $u_{0}$ be a discrete function $u_{0}=\left\{u_{0}^{1}, \ldots, u_{0}^{\mathrm{n}}\right\}$. For the function $u_{0}$ the problem in Eq. (1) takes following form:

$$
J(u)=\sum_{i=1}^{n}\left(u^{i}-u_{0}^{i}\right)^{2}+\lambda \sum_{i=1}^{n-1}\left|u^{i+1}-u^{i}\right| \text {. }
$$

The functional $J(u)$ is convex. Thus for the extremal (minimum) function $u_{*}$ the subgradient $\nabla J(u)$ satisfies the condition:

$$
\mathbf{0} \in \nabla J\left(u_{*}\right) \text {. }
$$

Remark. The subgradient $\nabla f(x)$ of the function $f(x)=|x|$ :

$$
\nabla f(x)=\left\{\begin{array}{c}
1, \text { if } x>0 \\
-1, x<0 \\
{[-1 ; 1], x=0}
\end{array}\right.
$$

\subsection{Computation of the subgradient}

Consider subgradient $\nabla J(u)$ :

$$
\begin{gathered}
\nabla J(u)=\sum_{i=1}^{n} \nabla\left(u^{i}-u_{0}^{i}\right)^{2}+\lambda \sum_{i=1}^{n-1} \nabla\left|u^{i+1}-u^{i}\right| . \\
\sum_{i=1}^{n} \nabla\left(u^{i}-u_{0}^{i}\right)^{2}=\left(u^{1}-u_{0}^{1}, u^{2}-u_{0}^{2}, \ldots, u^{1 n-1}-u_{0}^{n-1}, u^{n}-u_{0}^{n}\right) .
\end{gathered}
$$

In a similar manner with Eq. (6) the subgradients $\nabla\left|u^{i+1}-u^{i}\right|, i=1, \ldots, n-1$, can be written as 


$$
\nabla\left|u^{2}-u^{1}\right|=\left\{\begin{array}{c}
(-1,1,0,0,0, \ldots, 0,0), \text { if } u^{2}>u^{1} \\
(1,-1,0,0,0, \ldots, 0,0), \text { if } u^{2}<u^{1} \\
\left\{\left(\delta^{1},-\delta^{1}, 0,0,0, \ldots, 0,0\right) \mid \delta^{1} \in[-1 ; 1]\right\}, \text { if } u^{2}=u^{1}
\end{array}\right.
$$

$$
\nabla\left|u^{3}-u^{2}\right|=\left\{\begin{array}{c}
(0,-1,1,0,0, \ldots, 0,0), \text { if } u^{3}>u^{2} \\
(0,1,-1,0,0, \ldots, 0,0), \text { if } u^{3}<u^{2} \\
\left\{\left(0, \delta^{2},-\delta^{2}, 0,0, \ldots, 0,0\right) \mid \delta^{2} \in[-1 ; 1]\right\}, \text { if } u^{3}=u^{2}
\end{array}\right.
$$

$$
\nabla\left|u^{n-1}-u^{n-2}\right|=\left\{\begin{array}{c}
(0,0,0,0,0, \ldots,-1,1,0), \text { if } u^{n-1}>u^{n-2} \\
(0,0,0,0,0, \ldots, 1,-1,0), \text { if } u^{n-1}<u^{n-2} \\
\left\{\left(0,0,0,0,0, \ldots, \delta^{n-2},-\delta^{n-2}, 0\right) \mid \delta^{n-2} \in[-1 ; 1]\right\}, \text { if } u^{n-1}=u^{n-2}
\end{array},\right.
$$

$$
\nabla\left|u^{n}-u^{n-1}\right|=\left\{\begin{array}{c}
(0,0,0,0,0, \ldots 0,-1,1), \text { if } u^{n}>u^{n-1} \\
(0,0,0,0,0, \ldots 0,1,-1), \text { if } u^{n}<u^{n-1} \\
\left\{\left(0,0,0,0,0, \ldots, 0, \delta^{n-1},-\delta^{n-1}\right) \mid \delta^{n-1} \in[-1 ; 1]\right\}, \text { if } u^{n}=u^{n-1}
\end{array},\right.
$$

$\sum_{i=1}^{n-1} \nabla\left|u^{i+1}-u^{i}\right|=\left\{\left(\delta^{1}, \delta^{2}-\delta^{1}, \delta^{3}-\delta^{2}, \delta^{4}-\delta^{3}, \ldots, \delta^{n-1}-\delta^{n-2},-\delta^{n-1}\right) \mid \delta^{i}=-1\right.$, if $u^{i+1}>u^{i}, \delta^{i}=1$, if $u^{i+1}<u^{i}$,

$$
\left.\delta^{i} \in[-1 ; 1], \text { if } u^{i+1}=u^{i}, i=1, \ldots, n-1\right\} .
$$

From expressions (8) and (13) we get the following parameterization of the subradient:

$$
\left\{\begin{array}{c}
(\nabla J(u))^{1}=\left(u^{1}-u_{0}^{1}\right)+\lambda \delta^{1} \\
(\nabla J(u))^{2}=\left(u^{2}-u_{0}^{2}\right)+\lambda \delta^{2}-\lambda \delta^{1} \\
(\nabla J(u))^{3}=\left(u^{3}-u_{0}^{3}\right)+\lambda \delta^{3}-\lambda \delta^{2} \\
\cdots \\
(\nabla J(u))^{n-1}=\left(u^{n-1}-u_{0}^{n-1}\right)+\lambda \delta^{n-1}-\lambda \delta^{n-2} \\
(\nabla J(u))^{n}=\left(u^{n}-u_{0}^{n}\right)+\lambda \delta^{n-1}
\end{array}\right.
$$

where

$$
\delta^{i}=\left\{\begin{array}{c}
-1, \text { if } u^{i+1}>u^{i} \\
1, \text { if } u^{i+1}<u^{i} \\
\in[-1 ; 1], \text { if } u^{i+1}=u^{i}
\end{array} .\right.
$$

Since $\left(\nabla J\left(u_{*}\right)\right)^{i}=0, i=1, \ldots, n-1$ for some values of the parameters $\delta^{i}$ satisfying Eq. (15) we get:

$$
\left\{\begin{array}{c}
u_{*}^{1}=u_{0}^{1}-\lambda \delta^{1} \\
u_{*}^{2}=u_{0}^{2}-\lambda \delta^{2}+\lambda \delta^{1} \\
u_{*}^{3}=u_{0}^{3}-\lambda \delta^{3}+\lambda \delta^{2} \\
\cdots \\
u_{*}^{n-1}=u_{0}^{n-1}-\lambda \delta^{n-1}+\lambda \delta^{n-2} \\
u_{*}^{n}=u_{0}^{n}+\lambda \delta^{n-1}
\end{array} .\right.
$$

Consider the sequence of the cumulative sums:

$$
\left\{\begin{array}{c}
u_{*}^{1}=u_{0}^{1}-\lambda \delta^{1} \\
u_{*}^{2}+u_{*}^{1}=u_{0}^{2}+u_{0}^{1}-\lambda \delta^{2} \\
u_{*}^{3}+u_{*}^{2}+u_{*}^{1}=u_{0}^{3}+u_{0}^{2}+u_{0}^{1}-\lambda \delta^{3} \\
\cdots \\
u_{*}^{n-1}+\cdots+u_{*}^{1}=u_{0}^{n-1}+\cdots+u_{0}^{1}-\lambda \delta^{n-1} \\
u_{*}^{n}+\cdots+u_{*}^{1}=u_{0}^{n}+\cdots+u_{0}^{1}
\end{array} .\right.
$$

Consider such variables $U^{1}, \ldots, U^{n}$ and $U_{0}^{1}, \ldots, U_{0}^{n}$, that

$$
\left\{\begin{array}{c}
U^{1}=u_{*}^{1}, U_{0}^{1}=u_{0}^{1} \\
U^{2}=u_{*}^{2}+u_{*}^{1}, U_{0}^{2}=u_{0}^{2}+u_{0}^{1} \\
\cdots \\
U^{n-1}=u_{*}^{n-1}+\cdots+u_{*}^{1}, U_{0}^{n-1}=u_{0}^{n-1}+\cdots+u_{0}^{1} \\
U^{n}=u_{*}^{n}+\cdots+u_{*}^{1}, U_{0}^{n}=u_{0}^{n}+\cdots+u_{0}^{1}
\end{array} .\right.
$$


Image Processing, Geoinformation Technology and Information Security / A. Makovetskii, S. Voronin, V. Kober

So the solution to the problem in Eq. (3) is reduced to the solution of the problem:

$$
\left\{\begin{array}{c}
U^{1}=U_{0}^{1}-\lambda \delta^{1} \\
U^{2}=U_{0}^{2}-\lambda \delta^{2} \\
U^{3}=U_{0}^{3}-\lambda \delta^{3} \\
\cdots \\
U^{n-1}=U_{0}^{n-1}-\lambda \delta^{n-1} \\
U^{n}=U_{0}^{n}
\end{array},\right.
$$

with given discrete function $U_{0}$ and unknown discrete functions $U$ and $\delta$ satisfying to the conditions in Eq. (15).

Consider additional variables $U^{0}=U_{0}^{0}=0$. Note that then for any $i=1, \ldots, n-1$ the condition $u^{i+1}>u^{i}$ is equivalent to the condition $U^{i+1}-2 U^{i}+U^{i-1}>0$, the condition $u^{i+1}<u^{i}$ is equivalent to the condition $U^{i+1}-2 U^{i}+U^{i-1}<0$, the condition $u^{i+1}=u^{i}$ is equivalent to the condition $U^{i+1}-2 U^{i}+U^{i-1}=0$.

Then the set of equations in Eq. (19) can be rewritten taking into account additional variables:

$$
\left\{\begin{array}{c}
U^{0}=U_{0}^{0}=0 \\
U^{1}=U_{0}^{1}-\lambda \delta^{1} \\
U^{2}=U_{0}^{2}-\lambda \delta^{2} \\
U^{3}=U_{0}^{3}-\lambda \delta^{3} \\
\cdots \\
U^{n-1}=U_{0}^{n-1}-\lambda \delta^{n-1} \\
U^{n}=U_{0}^{n}
\end{array}\right.
$$

where

$$
\delta^{i}=\left\{\begin{array}{c}
-1, \text { if } U^{i+1}-2 U^{i}+U^{i-1}>0 \\
1, \text { if } U^{i+1}-2 U^{i}+U^{i-1}<0 \\
\in[-1 ; 1], \text { if } U^{i+1}-2 U^{i}+U^{i-1}=0
\end{array} .\right.
$$

\subsection{Construction the ,tube}

The values $U_{0}^{0}, U_{0}^{1}, \ldots, U_{0}^{n}$ of the discrete function $U_{0}$ defines a piecewise linear curve, which is an axial line of the tube. The values $U_{0}^{0}, U_{0}^{1}+\lambda, \ldots, U_{0}^{n-1}+\lambda, U_{0}^{n}$ form the upper piecewise linear border of the tube, the values $U_{0}^{0}, U_{0}^{1}-\lambda, \ldots, U_{0}^{n-1}-\lambda, U_{0}^{n}$ form the bottom piecewise linear border of the tube. Figure 1 shows an example of a tube.
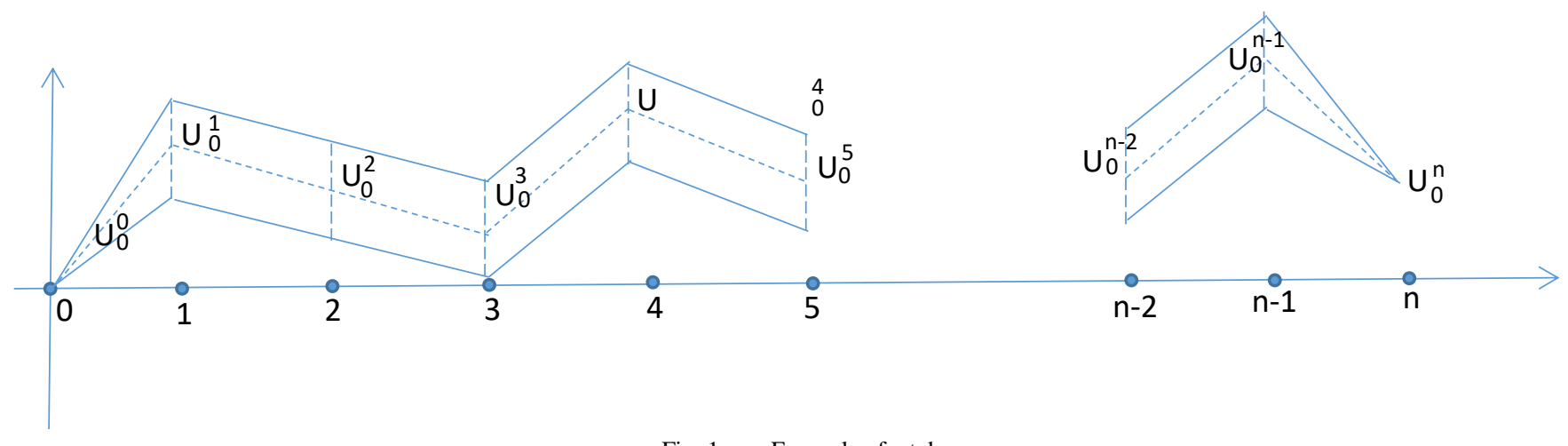

Fig. 1. Example of a tube.

\subsection{Description of the extremal function $U$}

Since $\delta^{\mathrm{i}}, \mathrm{i}=1, \ldots, \mathrm{n}-1$, take values in the segment $[-1 ; 1]$, a piecewise linear curve defined by the values $U^{1}, \ldots, U^{n}$ of a discrete function U (i.e. solution to the problem in Eq. (20)) entirely belongs to the tube.

If the second discrete derivative equals zero, $U^{i+1}-2 U^{i}+U^{i-1}=0$ then the piecewise linear curve defined by the values $\mathrm{U}^{1}, \ldots, \mathrm{U}^{\mathrm{n}}$ of a discrete function $\mathrm{U}$ in the neighborhood of the point $i$ is a straight line.

If the second discrete derivative is positive, $U^{i+1}-2 U^{i}+U^{i-1}>0$ then from Eq. (21) we see that $\delta^{i}=-1$ and Eq. (20) shows us that $U^{i}=U_{0}^{i}+\lambda$, i.e. $U^{i}$ belongs to the upper border of the tube.

If the second discrete derivative is negative, $U^{i+1}-2 U^{i}+U^{i-1}<0$ then from Eq. (21) we see that $\delta^{i}=1$ and Eq. (20) shows us that $U^{i}=U_{0}^{i}-\lambda$, i.e. $U^{i}$ belongs to the lower border of the tube.

It means that a piecewise linear curve defined by the values $\mathrm{U}^{0}, \ldots, \mathrm{U}^{\mathrm{n}}$ of a discrete function $\mathrm{U}$ exactly coincides with so called ,taut string" connecting the endpoints of the tube. 


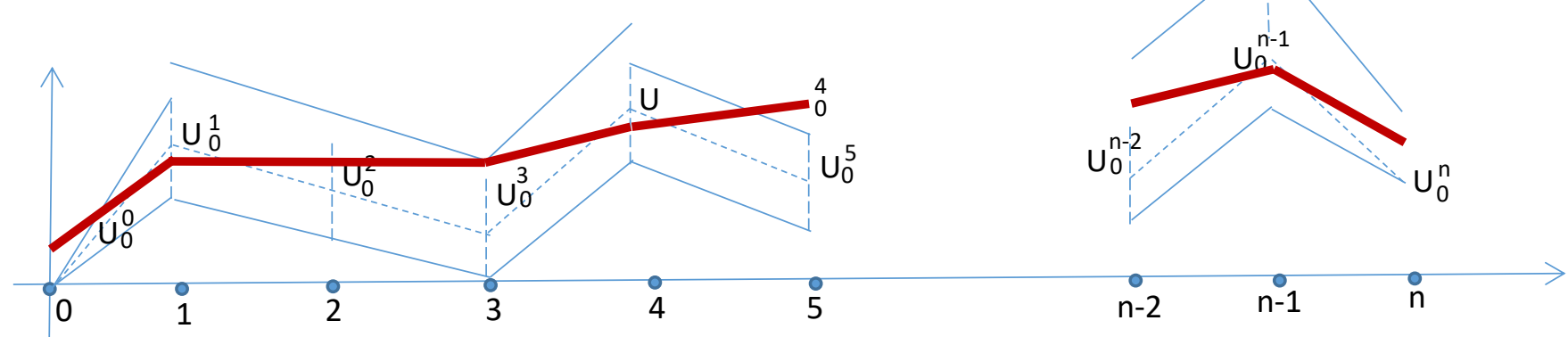

Fig. 2. Taut string in the tube.

\section{Conclusion}

In this paper, we propose a variant of the Condat's method based on the direct 1D TV regularization problem. The usage of the Condat'smethod with the taut string method leads to a clear geometric description of the extremal function.

\section{Acknowledgements}

The work was supported by Russian Science Foundation grant №15-19-10010.

\section{References}

[1] Rudin L, Osher S, Fatemi E. Nonlinear total variation based noised removal algorithms. Phys. D 1992; 60: 259-268.

[2] Chambolle A, Lions PL. Image recovery via total variational minimization and related problems. Numer. Math. 1997; 76: $167-188$.

[3] Osher S, Burger M, Goldfarb D, Xu J, Yin W. An iterative regularization method for total variation based image restoration. Multiscale Modelling and Simulation 2005; 4: 460-489.

[4] Chambolle A. An Algorithm for Total Variation Minimization and Applications. Journal of Mathematical Imaging and Vision 2004; 20 : 89-97.

[5] Strong DM, Chan TF. Exact Solutions to Total Variation Regularization Problems. UCLA CAM Report, 1996.

[6] Strong DM, Chan TF. Edge-preserving and scale-dependent properties of total variation regularization. Inverse Problems 2003; 19 : $165-187$.

[7] Voronin S, Makovetskii A, Kober V, Karnauhov V. Properties of exact solutions of the total variation regularization functions of one variable. Journal of Communications Technology and Electronics 2015; 60: 1356-1359.

[8] Voronin S, Makovetskii A, Kober V. Explicit solutions of one-dimensional total variation problem. Proc. SPIE's 60 Annual Meeting: Applications of Digital Image Processing XXXVIII 2015; 9599: 959926-1.

[9] Voronin S, Makovetskii A, Kober V. An efficient algorithm for total variation denoising. Proc. Int. Conference of Analysis of Images, Social Networks, and Texts (AIST) 2016; 236-248.

[10] Voronin S, Makovetskii A, Kober V. Total variation regularization with bounded linear variations. Proc. SPIE's 61 Annual Meeting: Applications of Digital Image Processing XXXIX 2016; 9971: 99712T-9.

[11] Condat L. A Direct Algorithm for 1-D Total Variation Denoising. IEEE Signal Processing Letters 2013; $20(11): 1054-1057$.

[12] Davies PL, Kovac A. Local extremes, runs, strings and multiresolution. Ann. Statist. 2001; 29(1): 1-65. 